\title{
Underactuated control in parallel transported directions: the example of the three dimensional Heisenberg group
}

\author{
Lance Drager, Jack L. Follis, and Jeffrey M. Lee
}

The authors consider a natural reachability problem on a Riemannian manifold. Given an initial point on a manifold together with an initial subspace of the tangent space at that point, consider piecewise smooth curves such that the velocity at each point along the curve is tangent to the parallel translation of the given initial subspace. The problem is to determine or characterize the set of points reachable by such curves. The authors show that the problem can be formulated in terms of the standard control theory machinery of singular distributions and vector fields by lifting to the frame bundle. It is shown that if the initial velocity subspace is tangent to a totally geodesic submanifold, then the reachable set is contained in that submanifold. Thus our problem makes contact with the existence and uniqueness problem for totally geodesic submanifolds.

In the absence of a general result along these lines, it is natural to consider special cases. The authors consider the case where $M$ is the three dimensional Heisenberg group. We show that in this example, all points are reachable and further, the final configuration of the subspace carried along can be specified as well. This stronger result will be expressed in terms of the orthonormal frame bundle.

\section{Introduction}

In this paper we consider a problem in control theory which is geometrically natural and is connected with geometric problems such as the existence of totally geodesic submanifolds (See [5] and [4]). After proving a few general results, we will work out the problem in the example of the three dimensional Heisenberg group with a left invariant metric. By working through this special case we wish to show that our formulation of this problem in terms of the theory of generalized tangent distributions is both theoretically natural and computationally feasible. 
Our problem can be formulated on a manifold with an arbitrary connection, but our example is not so general. For the general problem, let $M$ be a complete Riemannian manifold of dimension $m>1$. Suppose we are at the point $p$ in $M$ and we are flying some sort of craft that has controls on velocity that act in less than $m$ dimensions. Thus, our initial velocity vector would be confined to a subspace $V_{0} \subseteq T_{p} M$ of dimension $k<m$. As we move through $M$, how do the directions in which we have control evolve? A natural assumption is that they evolve inertially, i.e., by parallel translation. The question is: which points of $M$ can we reach?

To formulate the problem more precisely, given a point $p$ in $M$ and a subspace $V_{0} \subseteq T_{p} M$, we say a smooth curve $\gamma:[a, b] \rightarrow M$ is admissible for the initial data $\left(p, V_{0}\right)$ if $\gamma(a)=p$ and $\gamma^{\prime}(t) \in V_{t}$ for all $t$, where $V_{t}$ is the parallel translation of $V_{0}$ to $\gamma(t)$ along the curve $\gamma$. We can extend the definition to piecewise smooth curves by requiring this condition on each subinterval where the curve is smooth.

There is another interesting formulation. Let $u^{0}=\left(u_{1}^{0}, u_{2}^{0}, \ldots, u_{k}^{0}, u_{k+1}^{0}\right.$, $\ldots, u_{m}^{0}$ ) be an orthonormal frame (i.e., basis) for $T_{p} M$ so that $V_{0}=\operatorname{span}\left(u_{1}^{0}, \ldots, u_{k}^{0}\right)$. Then a smooth curve $\gamma$ is admissible if and only if there are real-valued functions $f^{1}, f^{2}, \ldots, f^{k}$ so that

$$
\gamma^{\prime}(t)=\sum_{i=1}^{k} f^{i}(t) u_{i}(t)
$$

where $u_{i}(t)$ is the parallel translation of the vector $u_{i}^{0}$ along $\gamma$. As we will see below, the functions $f^{i}$ can be thought of as controls.

Given the initial data $\left(p, V_{0}\right)$, we say a point $q \in M$ is reachable for this initial data if there is a piecewise smooth curve $\gamma:[a, b] \rightarrow M$ that is admissible for $\left(p, V_{0}\right)$ such that $\gamma(a)=p$ and $\gamma(b)=q$. The set of points that are reachable with initial data $\left(p, V_{0}\right)$ will be denoted by $R\left(p, V_{0}\right)$.

A couple of simple examples are evident. If $V_{0}$ has dimension 1 , a smooth admissible curve must be a reparametrization of a geodesic, and the image of the geodesic is a one dimensional totally geodesic immersed submanifold, so our problem is a generalization of the geodesic problem.

If $M$ is $m$-dimensional Euclidean space, the reachable set is the affine subspace $p+V_{0}$ determined by the initial data. More generally, if $M$ is a space of constant curvature, there is a totally geodesic submanifold $N$ so that $T_{p} N=V_{0}$, and the reachable set is $N$ [3, Chap. III, Lemma 1.1].

We will prove below that if $N$ is a complete totally geodesic submanifold of $M$ so that $V_{0} \subseteq T_{p} N \subseteq T_{p} M$, then an admissible curve must stay in $N$, so $R\left(p, V_{0}\right) \subseteq N$. 
Inspired by [15], [14] and [4] one might conjecture that $R\left(p, V_{0}\right)$ is the smallest complete totally geodesic immersed submanifold $N$ of $M$ such that $V_{0} \subseteq T_{p} N$. For an example where the reachable set is immersed but not imbedded, consider a line of irrational slope on a flat torus, which is a geodesic and a dense immersed submanifold. As far as we know, this conjecture might be true if we work locally. Globally, things must be more complicated. After all, a special case is the image of a geodesic and geodesics can cross themselves (consider a cone). In the higher dimensional case there may be more complex self intersection and perhaps singularities. The source of these phenomena and an approach to analyze them will be clearer below. Of course, none of these phenomena occur in the current example.

In our example, we take the ambient manifold $M$ to be the three dimensional Heisenberg group $H^{3}$. We choose this space because it is easy to visualize, it does not have constant curvature, it is an important example in the related area of subriemannian geometry, and because everything can be explicitly calculated.

We will show that the reachable set $R\left(p, V_{0}\right)$ is all of $H^{3}$ for arbitrary initial data with $\operatorname{dim}\left(V_{0}\right)=2$.

We will also consider, not just the reachable points in $M$ but also ask about the final result of parallel translating the initial velocity subspace. Indeed, it is natural to ask for even more. If we are given a frame for the tangent space at the initial point whose first $k$ members span the initial velocity subspace, then we parallel translate the frame along admissible curves and ask about the possible final frame at the endpoint. Thus we are led to ask for a reachable set on the level of the orthonormal frame bundle. If we adopt the imagery of a craft moving through the manifold and to which the parallel translated initial velocity subspace is rigidly fixed, then we are asking about the possibility of arriving with the craft in any prescribed attitude.

\section{Lifting the problem to the frame bundle}

As stated, our problem is not phrased in terms vector fields and distributions, the usual apparatus for nonlinear control theory. We can rephrase the problem in this form if we lift the problem to the bundle of orthonormal frames. We very briefly recall the apparatus of connections on frame bundles, mostly to fix our conventions and notation. Some standard references are $[6,10]$.

Let $M$ be our complete Riemannian manifold. An orthonormal frame for the tangent space $T_{p} M$ is just an orthonormal basis $u=\left(u_{1}, \ldots, u_{m}\right)$ of this 
inner product space. We will identify the frame $u$ with the linear isometry $\mathbb{R}^{m} \rightarrow T_{p} M$ that sends the $i$-th standard basis vector $e_{i}$ to $u_{i}$. Let $F_{p}(M)$ denote the set of orthonormal frames of $T_{p} M$. The orthogonal group $\mathrm{O}(m)$ of orthogonal $m \times m$ matrices acts on $F_{p}(M)$ on the right. If $u \in F_{p}(M)$ and $g \in \mathrm{O}(m)$, then $u \cdot g$ can be thought of as the composition of $u: \mathbb{R}^{m} \rightarrow T_{p} M$ and the map $\mathbb{R}^{m} \rightarrow \mathbb{R}^{m}$ given by multiplication by $g$ or, alternatively as the row vector $\left[u_{1}, \ldots, u_{m}\right]$ multiplied by the matrix $g$.

Let $F(M)$ be the union of the sets $F_{p}(M)$ for $p \in M$. There is a projection $\pi: F(M) \rightarrow M$ given by $\pi\left(F_{p}(M)\right)=p$. The fibration $\pi: F(M) \rightarrow M$ is a principal fiber bundle with structure group $\mathrm{O}(m)$.

If $u \in F(M)$, the tangent space at $u$ of the fiber through $u$ is the vertical space at $u$, denoted by $\mathcal{V}_{u}$. It can also be described as the kernel of $T \pi=$ $\pi_{*}: T_{u} F(M) \rightarrow T_{\pi(u)} M$. There is a linear isomophism $A \mapsto \mathcal{V}_{u}(A)$ from the Lie algebra $\mathfrak{s o}(m)$ to $\mathcal{V}_{u}$, defined by

$$
\mathcal{V}_{u}(A)=\left.\frac{d}{d t} u \cdot \exp (t A)\right|_{t=0} .
$$

On $F(M)$, we have an $\mathbb{R}^{m}$-valued one-form $\widetilde{\theta}$, called the solder form. The map $\widetilde{\theta}_{u}: T_{u} F(M) \rightarrow \mathbb{R}^{m}$ is defined by

$$
\widetilde{\theta}_{u}(X)=u^{-1}\left[\pi_{*}(X)\right]
$$

In other words, we project $X$ to the base with $\pi_{*}$, and then take the components of this element of $T_{\pi(u)} M$ with respect to the frame $u$. The solder form has the group invariance property

$$
R_{g}^{*} \widetilde{\theta}=g^{-1} \widetilde{\theta}
$$

where $R_{g}: F(M) \rightarrow F(M): u \mapsto u \cdot g$ is right multiplication by $g \in \mathrm{O}(m)$.

The Levi-Civita connection defined by the Riemannian metric can be described by its connection one form $\widetilde{\omega}$ on $F(M)$. This form takes values in the Lie Algebra $\mathfrak{s o}(m)$. It has the following action on the vertical spaces

$$
\widetilde{\omega}\left(\mathcal{V}_{u}(A)\right)=A
$$

and the group invariance property

$$
R_{g}^{*} \widetilde{\omega}=\operatorname{Ad}\left(g^{-1}\right) \widetilde{\omega}, \quad g \in \mathrm{O}(m) .
$$

Since we are dealing with a matrix group, the map $\operatorname{Ad}(g): \mathfrak{s o}(m) \rightarrow \mathfrak{s o}(m)$ is just conjugation $A \mapsto g A g^{-1}$. 
The kernel of the mapping $\widetilde{\omega}_{u}: T_{u} F(M) \rightarrow \mathfrak{s o}(m)$ is denoted by $\mathcal{H}_{u}$ and is called the horizontal subspace at $u$. For each $u$, we have $T_{u} F(M)=$ $\mathcal{H}_{u} \oplus \mathcal{V}_{u}$. Thus, $\pi_{*}$ maps $\mathcal{H}_{u}$ bijectively to $T_{\pi(u)} M$.

Let $\gamma:[a, b] \rightarrow M$ be a smooth curve, let $p=\gamma(a)$ and let $u^{0} \in F_{p}(M)$. Then there is a unique horizontal lift $\tilde{\gamma}$ of $\gamma$ starting at $u^{0}$. In other words, there is a unique curve $\tilde{\gamma}$ such that $\pi \circ \tilde{\gamma}=\gamma, \tilde{\gamma}(a)=u^{0}$ and $\tilde{\gamma}^{\prime}(t) \in \mathcal{H}_{\tilde{\gamma}(t)}$. Thought of as a frame, $\tilde{\gamma}(t)$ is the frame for $T_{\gamma(t)} M$ obtained by parallel translation of the frame $u^{0}$.

We will also need to use the basic horizontal vector fields. Given $\xi \in \mathbb{R}^{m}$, there is a unique vector field $H(\xi)$ on $F(M)$, the basic horizontal vector field for $\xi$, that satisfies the conditions

1. $\widetilde{\omega}(H(\xi))=0$.

2. $\widetilde{\theta}(H(\xi))=\xi$.

The first condition just says $H(\xi)$ is horizontal. The second condition means that $u^{-1}\left[\pi_{*} H_{u}(\xi)\right]=\xi$, so $H_{u}(\xi)$ is the vector in $\mathcal{H}_{u}$ that projects to the vector $u(\xi) \in T_{\pi(u)} M$. Thus, the map $\mathbb{R}^{m} \rightarrow \mathcal{H}_{u}: \xi \mapsto H_{u}(\xi)$ is a linear isomophism. If we let $H_{i}$ denote $H\left(e_{i}\right)$, we have

$$
H_{u}(\xi)=\sum_{i=1}^{m} \xi^{i} H_{i u}
$$

We now apply this machinery to our problem. We first consider the problem of control in parallel translated directions.

Theorem 1. Let $p$ be a point in $M$ and let $u^{0}$ be a frame for $T_{p} M$. Then, given smooth functions $f^{1}, \ldots, f^{m}:[a, b] \rightarrow \mathbb{R}$, there is a unique curve $\gamma:[a, b] \rightarrow M$ such that $\gamma(a)=p$ and

$$
\gamma^{\prime}(t)=\sum_{i=1}^{m} f^{i}(t) u_{i}(t)
$$

where $u_{i}(t)$ is the parallel translation of $u_{i}^{0}$ along $\gamma$.

Proof. Let $f$ be the $\mathbb{R}^{m}$-valued function $f(t)=\left(f^{1}(t), \ldots, f^{m}(t)\right)$. Suppose that the curve in the Proposition exists. Let $\tilde{\gamma}$ be the horizontal lift of $\gamma$ starting at $u^{0}$. Thought of as a frame, $\tilde{\gamma}(t)=u(t)=\left(u_{1}(t), \ldots, u_{m}(t)\right)$, the parallel transport of $u^{0}$ along $\gamma$. From (1),

$$
\pi_{*} \tilde{\gamma}^{\prime}(t)=\gamma^{\prime}(t)=\sum_{i=1}^{m} f^{i}(t) u_{i}(t)=u(t)(f(t)),
$$

so $\widetilde{\theta}\left(\tilde{\gamma}^{\prime}(t)\right)=f(t)$. Thus, $\tilde{\gamma}^{\prime}(t)=H_{\tilde{\gamma}(t)}(f(t))$. 
We can define a time dependent vector field on $F(M)$ by $(u, t) \mapsto$ $H_{u}(f(t))$. Our calculation shows that $\tilde{\gamma}$ is the unique integral curve of this vector field with initial value $u^{0}$ at $t=a$. Conversely, given the initial data $p$ and $u^{0}$, we can take $\tilde{\gamma}$ to be the integral curve of $H(f(t))$ with initial value $u^{0}$ at $t=a$, and then $\gamma=\pi \circ \tilde{\gamma}$ will be the curve that satisfies (1). The existence of this integral curve over the entire time interval $[a, b]$ follows from a simple compactness argument.

We can now prove our theorem about totally geodesic submanifolds.

Theorem 2. Let $M$ be a complete Riemannian manifold, let $p$ be a point in $M$ and let $V_{0} \subseteq T_{p} M$ be a subspace. Suppose that $N \subseteq M$ is a complete immersed totally geodesic submanifold of $M$ containing $p$ such that $V_{0} \subseteq$ $T_{p} N \subseteq T_{p} M$. Then any admissible curve for the initial data $\left(p, V_{0}\right)$ stays in $N$. Thus, $R\left(p, V_{0}\right) \subseteq N$.

Proof. Let $n=\operatorname{dim}(N)$ and $k=\operatorname{dim}\left(V_{0}\right)$. Choose a frame $u^{0}=\left(u_{1}^{0}, \ldots, u_{m}^{0}\right)$ such that $V_{0}=\operatorname{span}\left(u_{1}^{0}, \ldots, u_{k}^{0}\right)$ and $T_{p} N=\operatorname{span}\left(u_{1}^{0}, \ldots u_{n}^{0}\right)$.

If $\gamma$ is an admissible curve for initial data $\left(p, V_{0}\right)$, we can write

$$
\gamma^{\prime}(t)=\sum_{i=1}^{k} f^{i}(t) u_{i}(t)
$$

for some functions $f^{i}$, where $u_{i}(t)$ is the parallel translate of $u_{i}^{0}$ along $\gamma(t)$ with respect to the connection on $M$.

On the other hand, considering the problem in $N$, we can use the initial data $p$ and the frame $\left(u_{1}^{0}, \ldots, u_{n}^{0}\right)$, and the control functions $f^{i}$ to construct a curve $\bar{\gamma}$ in $N$ such that

$$
\bar{\gamma}^{\prime}(t)=\sum_{i=1}^{k} f^{i}(t) \bar{u}_{i}(t),
$$

where $\bar{u}_{i}(t)$ is the parallel translate of $u_{i}^{0}$ along $\bar{\gamma}$ with respect to the induced connection on $N$. But, $N$ is totally geodesic, so parallel translation of vectors tangent to $N$ along a curve in $N$ is the same with respect to both connections. Thus, we can rewrite (2) as

$$
\bar{\gamma}^{\prime}(t)=\sum_{i=1}^{k} f^{i}(t) u_{i}(t),
$$

where the $u_{i}$ 's are the parallel transports in $M$. By the uniqueness part of the last theorem, $\gamma=\bar{\gamma}$, so $\gamma$ is contained in $N$. 


\section{Distributions, brackets and reachable sets}

We can use the frame bundle machinery to reduce our problem to finding the set of points reachable by curves tangent to a distribution. This machinery is familiar in control theory, but may be new to some, so we include a brief discussion. It should be mentioned that we are considering singular distributions in that the dimension need not be locally constant. The basic results go back to Chow [1] and Nagano [9] and were later generalized by Sussmann [12] and Stefan [11]. A good modern exposition is given in [8, Section 3].

Let $N$ be any smooth manifold. A distribution is a function $p \mapsto E_{p}$ that assigns to each $p \in N$ a subspace $E_{p} \subseteq T_{p} N$ whose dimension may vary with $p$.

Let $\mathcal{X}_{\text {loc }}$ be the set of smooth vector fields whose domains are open subsets of $N$. Given $\mathcal{W} \subseteq \mathcal{X}_{\text {loc }}$, we can define a distribution $E(\mathcal{W})$ by

$$
E_{p}(\mathcal{W})=\operatorname{span}\left\{X_{p} \mid X \in \mathcal{W}, p \in \operatorname{dom}(X)\right\} .
$$

A distribution is smooth if it is spanned locally by smooth vector fields, i.e., if it is $E(\mathcal{W})$ for some set $\mathcal{W}$ of local vector fields. We say a locally defined vector field $X$ belongs to $E$ if $X_{p} \in E_{p}$ for all $p$ in the domain of $X$.

We mention in passing that it has recently been shown in [2] and [13] that for any such smooth singular distribution $E(\mathcal{W})$, there is a finite family $\mathcal{W}_{0}$ of smooth globally defined vector fields such that $E(\mathcal{W})=E\left(\mathcal{W}_{0}\right)$. Thus, in a limited sense, every singular distribution is finitely generated. However, it should be noted that space of smooth sections of such distributions need not be finitely generated as a module over the ring of smooth functions.

A distribution $E$ is integrable if each point of $N$ is contained in an integral manifold of $E$, i.e., for every $p \in N$ there is an immersed submanifold $S$ so that $S \ni p$ and $T_{q} S=E_{q}$ for all $q \in S$. It follows that each point is contained in a maximal connected integral submanifold. These maximal integral manifolds partition $N$, and form the leaves of a singular foliation of $N$. This means that for each leaf $L$ and each point $p$ in $L$, we can find a coordinate chart $(U, x)$ centered at $p$ so that each component of $L \cap U$ is a coordinate slice. There are countably many such components. In particular, such a submanifold is weakly imbedded [7].

Let $E=E(\mathcal{W})$ be a smooth distribution. We say that a smooth curve $\gamma:[a, b] \rightarrow N$ is admissible with respect to $E$ if $\gamma^{\prime}(t) \in E_{\gamma(t)}$ for all $t \in[a, b]$. We may say that $\gamma$ is tangent to the distribution $E$. This definition extends to piecewise smooth curves by requiring it to hold on each smooth piece. Let 


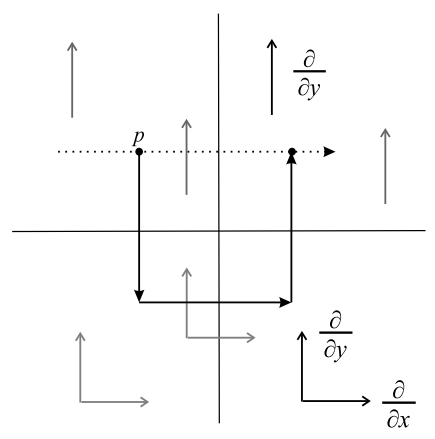

Figure 1: Line of reachable points but not an admissible curve.

$p$ be a point in $N$. We say that a point $q \in N$ is reachable from $p$ if there is an admissible curve $\gamma:[a, b] \rightarrow N$ so that $\gamma(a)=p$ and $\gamma(b)=q$. Given $p$, we want to determine the set of points that are reachable from $p$. One way to get admissible curves is to follow (forwards or backwards) integral curves of the vector fields that belong to the distribution. For a vector field $Z$, let $\left\{\Phi_{t}^{Z}\right\}$ be the flow of of $Z$.

One should distinguish carefully between the problem of finding the reachable set and the problem of finding a specific admissible curve between two specified points. The latter problem goes under the name of motion planning, which we do not enter into here.

To get an idea of what is going on, let us consider a simple example. Let the manifold $N$ be $\mathbb{R}^{2}$ with coordinates $(x, y)$. Let $Y$ be the globally defined vector field $Y=\partial / \partial y$. Let $U$ be the open lower half-plane, and let $X$ be the vector field $\partial / \partial x$ restricted to $U$. Let $\mathcal{W}=\{X, Y\}$ and $E=E(\mathcal{W})$. Thus, $E_{p}$ has dimension 1 for $p$ in the closed upper half-plane and dimension 2 for $p$ in the open lower half plane. The distribution is not integrable. For example, an integral manifold through the origin would have to be both one dimensional and two dimensional.

Let $p=(-1,1)$ and $q=(1,1)$. We claim that $q$ is reachable from $p$. To construct an admissible curve, follow the integral curve of $Y$ through $(-1,1)$ backwards for two units of time to reach $(-1,-1)$. From $(-1,-1)$ follow the integral curve of $X$ forward for two units of time to reach $(1,-1)$. Now follow the integral curve of $Y$ forward for two units of time to reach $q=(1,1)$. To put it another way, $q=\Phi_{2}^{Y} \Phi_{2}^{X} \Phi_{-2}^{Y}(p)$. See Figure 1 . By an obvious generalization of this construction, we see that all points in the plane can be reached from $p$ !

Define a curve $\gamma(t)=\Phi_{2}^{Y} \Phi_{t}^{X} \Phi_{-2}^{Y}(p)$ (the dotted line in the figure). Then $\gamma(0)=p$ and $\gamma(2)=q$. By our construction, all of the points $\gamma(t)$ are 
reachable, even though $\gamma$ itself is not an admissible curve. Thus, $\gamma$ is a curve in the reachable set. If we believe that the reachable set is some kind of submanifold, then $\gamma^{\prime}(0)$ must be tangent to it (so the reachable set must be two dimensional). Clearly we could not see this from local information at $p$. The vector $\gamma^{\prime}(0)$ is, by definition, the value at $p$ of the vector field $\left(\Phi_{-2}^{Y}\right)^{*} X$, the pullback of $X$ by the flow of $Y$.

This example hints at the general approach. We can not, in general, determine the reachable set from local information (such as differentiation). To find directions tangent to the reachable set, we should consider pullbacks of vector fields tangent to the distribution at other points.

The general construction goes as follows. Let $E=E(\mathcal{W})$ be a smooth distribution. A set $\mathcal{V}$ of locally defined vector fields is stable if whenever $X$ and $Y$ belong to $\mathcal{V}$ and $\left(\Phi_{t}^{X}\right)^{*} Y$ is defined on a nonempty open set, it belongs to $\mathcal{V}$. Let $\mathcal{S}(\mathcal{W})$ denote the smallest stable set of vector fields containing $\mathcal{W}$. Let $E^{*}=E(\mathcal{S}(\mathcal{W}))$, which can also be described as the smallest distribution containing $E$ (i.e., $E_{p} \subseteq E_{p}^{*}$ for all $p$ ) that is invariant under the flows of the vector fields in $\mathcal{W}$.

The main result in this setting is the following. The distribution $E^{*}$ is integrable, and is the smallest integrable distribution containing $E$. (Note that even if $E$ has constant dimension, $E^{*}$ may not.) Fix a point $p \in N$. The set of points that can be reached from $p$ by (piecewise) smooth curves that are tangent to $E$ is precisely the leaf $L(p)$ of the singular foliation determined by of $E^{*}$ that contains $p$. If $E_{q}^{*}=T_{q} N$ for all $q$, the leaf is $N$ itself, and all points can be reached from $p$.

Of course, $E^{*}$ is generally difficult to compute. A standard approach in control theory is to consider brackets of vector fields which belong to $E$, which can be computed by differentiation. These arise if we differentiate pullback vector fields with respect to the $t$-parameter, but we can get the idea from the usual commutator picture of the bracket. Suppose that $X$ and $Y$ are vector fields that belong to $E$ and that $p$ is a point. Define a curve by $\alpha(t)=\Phi_{-t}^{Y} \Phi_{-t}^{X} \Phi_{t}^{Y} \Phi_{t}^{X}(p)$, (see Figure 2). Again, each of the points $\alpha(t)$ is reachable from $p$, even though $\alpha$ is not itself an admissible curve. Thus, $\alpha$ lies in $L(p)$. As described in any introductory differential geometry book (See [7] page 107-108), $\alpha^{\prime}(0)=0$, so $\alpha^{\prime \prime}(0)$ can be considered to be a tangent vector. Since $\alpha$ lies in the submanifold $L(p), \alpha^{\prime \prime}(0) \in T_{p} L(p)$. But, $\alpha^{\prime \prime}(0)=2[X, Y]_{p}$. Thus, $[X, Y]_{p}$ is tangent to $L(p)$. If we are using two vector fields that we already know are tangent to the reachable set, the points on $\alpha$ will all be reachable, so the bracket will again be tangent to the reachable set.

Thus, we can find some additional directions tangent to the leaves of the foliation by taking brackets. As our example above shows, we will not in general get enough directions to find $E^{*}$. 


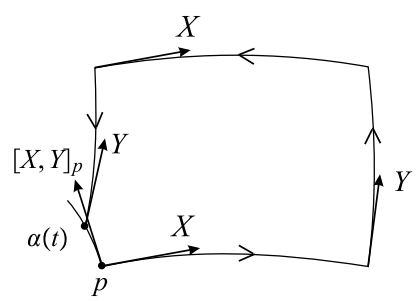

Figure 2: Commutator of Flows.

A distribution $F$ is involutive if the bracket of any two vector fields belonging to $F$ belongs to $F$ (the bracket of two locally defined vector fields is defined on the intersection of the domains). Our example above shows that an involutive singular distribution need not be integrable. A set $\mathcal{V}$ of vector fields is involutive if the bracket of any two vector fields in $\mathcal{V}$ is again in $\mathcal{V}$

If $E=E(\mathcal{W})$, let $\mathcal{L}(\mathcal{W})$ be the smallest set of vector fields containing $\mathcal{W}$ that is closed under taking brackets. Then $E \subseteq E(\mathcal{L}(\mathcal{W}))$ and $E(\mathcal{L}(\mathcal{W}))$ is the smallest involutive distribution containing $E$. In general we have

$$
E=E(\mathcal{W}) \subseteq E(\mathcal{L}(\mathcal{W})) \subseteq E(\mathcal{S}(\mathcal{W}))=E^{*}
$$

and the inclusions may be strict. The following standard theorem tells use when it will suffice to compute brackets. See [8].

Proposition 3. Consider distributions on a manifold $N$.

(1) If $\mathcal{V}$ is an involutive set of vector fields then $E(\mathcal{V})$ is integrable if and only if the dimension of $E(\mathcal{V})$ is constant along the integral curves of the vector fields in $\mathcal{V}$.

(2) If $N$ is real analytic and the involutive set $\mathcal{V}$ consists of globally defined analytic vector fields, then $E(\mathcal{V})$ is integrable.

(3) If $E=E(\mathcal{W})$, then $E(\mathcal{L}(\mathcal{W}))=E^{*}$ if and only if $E(\mathcal{L}(\mathcal{W}))$ is integrable.

(4) In particular, if $E(\mathcal{L}(\mathcal{W}))_{p}=T_{p} N$ at all points $p$, then the reachable set for any point is the whole manifold $N$.

Note that the first statement also implies the standard Frobenius Theorem.

To apply this machinery to our problem, let $M$ be a complete Riemannian manifold of dimension $m$, let $p$ be a point in $M$ and let $V_{0} \subseteq T_{p} M$ be a subspace of dimension $k$. Choose a frame $u^{0}$ so that $V_{0}=\operatorname{span}\left(u_{1}^{0}, \ldots, u_{k}^{0}\right)$. 
Let $E$ be the distribution on $F(M)$ spanned by the basic horizontal vector fields $H_{1}, \ldots, H_{k}$.

Lemma 4. A curve $\gamma:[a, b] \rightarrow M$ with $\gamma(a)=p$ is admissible for the initial data $\left(p, V_{0}\right)$ if and only if the horizontal lift $\tilde{\gamma}$ of $\gamma$ with $\tilde{\gamma}(a)=u^{0}$ is tangent to the distribution $E$.

Proof. If $\gamma$ is admissible, there are functions $f^{1}, \ldots, f^{k}$ so that

$$
\gamma^{\prime}(t)=\sum_{i=1}^{k} f^{i}(t) u_{i}(t)
$$

where $u(t)=\tilde{\gamma}(t)$ is the parallel transport of $u^{0}$ along $\gamma$. Let $f$ be the $\mathbb{R}^{m}$-valued function $f(t)=\left(f^{1}(t), \ldots, f^{k}(t), 0, \ldots, 0\right)$.

As we saw above, (3) is equivalent to saying $\tilde{\gamma}$ is an integral curve of the time dependent vector field $H(f(t))$. But then

$$
\tilde{\gamma}^{\prime}(t)=H_{u(t)}(f(t))=\sum_{i=1}^{m} f^{i}(t) H_{i u(t)}=\sum_{i=1}^{k} f^{i}(t) H_{i u(t)} \in E
$$

since the last $m-k$ components of $f$ are zero. Conversely if $\tilde{\gamma}$ is the integral curve of $H(f(t))$ starting at $u^{0}$ at $t=a$, then (4) holds, so $\tilde{\gamma}$ is tangent to $E$. Applying $\pi_{*}$ to $\tilde{\gamma}^{\prime}(t)$ shows that (3) holds, so $\gamma$ is admissible.

Thus, we have the following procedure for finding the reachable set $R\left(p, V_{0}\right)$. First, find the smallest integrable distribution $E^{*}$ on the frame bundle that contains the distribution $E$ spanned by $H_{1}, \ldots, H_{k}$. Given initial data $\left(p, V_{0}\right)$, choose a frame $u^{0}$ adapted to the initial data, and then find the maximal integral manifold $S$ of $E^{*}$ that passes through $u^{0}$. Then $R\left(p, V_{0}\right)=\pi(S)$.

This shows the source of the self-crossings and possible singularities in the reachable set. The leaf $S$ itself is an immersed, weakly imbedded submanifold of the frame bundle. If $S$ crosses the fiber of the frame bundle above $q$ and then comes back later to cross it again, the reachable set will have a self intersection. For example, the horizontal lift of a geodesic is an integral curve of a basic horizontal vector field and so does not cross itself. But the geodesic, the projection into the base, may cross itself. Since the bracket of horizontal vector fields is vertical, $T \pi$ will generally have a kernel in $T_{u} S$, so $\left.\pi\right|_{S}$ will usually not be an immersion of $S$ into $M$, thus there may be singularities in $\pi(S)$. To find out what happens, we need to study $S$ and its tangent spaces. Fortunately, none of these complexities arise in the case of the three dimensional Heisenberg group. 


\section{Computing in a global trivialization}

In this section, we work out how to express the condition that a vector field on the frame bundle of a parallelizable Riemannian manifold be horizontal in terms of a trivialization given by a fixed global frame field. In our example, there is a natural orientation, so we will work in the oriented frame bundle, denoted by $F(M)$ again.

Suppose that $M$ is a smooth oriented Riemannian manifold of dimension $m$ whose oriented orthonormal frame bundle $F(M)$ has a global smooth section $\sigma: M \rightarrow F(M)$. In what follows, let $G$ denote $\mathrm{SO}(m)$ and write $\mathfrak{s o}(m)$ as $\mathfrak{g}$. We view $G$ as the group of orthogonal matrices of determinant 1 whenever convenient. Then we have a diffeomorphism

$$
\Psi: M \times G \rightarrow F(M)
$$

given by $(p, g) \mapsto \sigma(p) g$. This map is also a principal bundle isomophism if we give $M \times G \rightarrow M$ the product principal bundle structure. We want to transfer all relevant structures to $M \times G$ for computational convenience. In particular, we effectively transfer the connection on $F(M)$ to a connection on $M \times G$.

Of course, the tangent space $T_{(p, g)}(M \times G)$ can be identified with $T_{p} M \oplus$ $T_{g} G$. For each $X_{p} \in T_{p} M$, we define its lift $X_{(p, g)} \in T_{(p, g)}(M \times G)$. If $c$ is a curve in $M$ with $c^{\prime}(0)=X_{p}$ then $X_{(p, g)}=\widetilde{c}^{\prime}(0)$ where $\widetilde{c}(t)=(c(t), g)$. Similarly, for any $Y_{g} \in T_{g} G$ we have the lift $Y_{(p, g)} \in T_{(p, g)}(M \times G)$. All elements of $T_{(p, g)}(M \times G)$ can be decomposed as $X_{(p, g)}+Y_{(p, g)}$ for appropriate $X_{p}$ and $Y_{g}$. We wish to discover the conditions for $X_{(p, g)}+Y_{(p, g)}$ to be horizontal. For this we push forward to $F(M)$. In what follows, let $\mu: T G \rightarrow$ $\mathfrak{g}$ be the right Maurer-Cartan form on $G$ defined by $\mu\left(Y_{g}\right)=\left(R_{g^{-1}}\right)_{*} Y_{g}$ where $R_{g^{-1}}: a \mapsto a g^{-1}$. Let us first compute $\Psi_{*} X_{(p, g)}$ where $X_{(p, g)}$ is the lift of some $X_{p} \in T_{p} M$. With $c$ and $\widetilde{c}$ as above, we have

$$
\begin{aligned}
\Psi_{*} X_{(p, g)} & =\left.\frac{d}{d t} \Psi(\widetilde{c}(t))\right|_{t=0}=\left.\frac{d}{d t} \Psi(c(t), g)\right|_{t=0} \\
& =\left.\frac{d}{d t} \sigma(c(t)) g\right|_{t=0}=\left.\frac{d}{d t}\left(R_{g} \circ \sigma \circ c\right)(t)\right|_{t=0} \\
& =R_{g *} \sigma_{*} X_{p} .
\end{aligned}
$$

Next let $Y_{(p, g)}$ be the lift of some $Y_{g} \in T_{g} G$. For $\gamma$ a curve with $\gamma^{\prime}(0)=Y_{g}$, we let $\widetilde{\gamma}$ be given by $\widetilde{\gamma}(t)=(p, \gamma(t))$ so that $Y_{(p, g)}=\widetilde{\gamma}^{\prime}(0)$. We can take $\gamma$ 
to be $\gamma(t)=\exp \left(t \mu\left(Y_{g}\right)\right) g$. We have

$$
\begin{aligned}
\Psi_{*} Y_{(p, g)} & =\left.\frac{d}{d t} \Psi\left(p, e^{t \mu\left(Y_{g}\right)} g\right)\right|_{t=0}=\left.\frac{d}{d t} \sigma(p) e^{t \mu\left(Y_{g}\right)} g\right|_{t=0} \\
& =\left.\frac{d}{d t} R_{g}\left(\sigma(p) e^{t \mu\left(Y_{g}\right)}\right)\right|_{t=0}=\left(R_{g}\right)_{*} \mathcal{V}\left(\mu\left(Y_{g}\right)\right)_{\sigma(p)}
\end{aligned}
$$

For any $Y \in \mathfrak{g}$, we have $\left(R_{g}\right)_{*} \mathcal{V}(Y)_{u}=\mathcal{V}\left(\operatorname{Ad}\left(g^{-1}\right) Y\right)_{u g}$. Indeed,

$$
\begin{aligned}
\left(R_{g}\right)_{*} \mathcal{V}(Y)_{u} & =\left.\frac{d}{d t} R_{g}\left(u e^{t Y}\right)\right|_{t=0}=\left.\frac{d}{d t} u e^{t Y} g\right|_{t=0} \\
& =\left.\frac{d}{d t} u g g^{-1} e^{t Y} g\right|_{t=0}=\left.\frac{d}{d t}(u g) e^{t \operatorname{Ad}\left(g^{-1}\right) Y}\right|_{t=0} \\
& =\mathcal{V}\left(\operatorname{Ad}\left(g^{-1}\right) Y\right)_{u g} .
\end{aligned}
$$

Thus

$$
\Psi_{*}\left(Y_{(p, g)}\right)=\mathcal{V}\left(\operatorname{Ad}\left(g^{-1}\right) \mu(Y)\right)_{\Psi(p, g)} .
$$

We conclude that a typical vector in $T_{u} F(M)$, where $u=\Psi(p, g)$, can be expressed as

$$
R_{g *} \sigma_{*}\left(X_{p}\right)+\mathcal{V}\left(\operatorname{Ad}\left(g^{-1}\right) \mu\left(Y_{g}\right)\right)_{\Psi(p, g)} .
$$

Let $\widetilde{\omega}$ denote the Levi-Civita connection form on $F(M)$ and $\widetilde{\theta}$ denote the solder form.

In order to take advantage of our global trivialization, we compute $\Psi^{*} \widetilde{\omega}$ and $\Psi^{*} \widetilde{\theta}$. We use the decomposition discovered above. For $\Psi^{*} \widetilde{\theta}$, we first note that for any $X_{p} \in T_{p} M$ we have $\sigma(p)^{-1}\left(X_{p}\right)=\theta\left(X_{p}\right)$ where $\theta$ is the column vector $\left[\theta^{1}, \ldots, \theta^{d}\right]^{\top}$ of 1 -forms dual to the frame field $\left(X_{1}, \ldots, X_{m}\right)$ determined by $\sigma$. We have

$$
\begin{aligned}
\left(\Psi^{*} \widetilde{\theta}\right)\left(X_{(p, g)}+Y_{(p, g)}\right) & =\widetilde{\theta}\left(R_{g *} \sigma_{*}\left(X_{p}\right)+\mathcal{V}\left(\operatorname{Ad}\left(g^{-1}\right) \mu\left(Y_{g}\right)\right)\right. \\
& =g^{-1} \widetilde{\theta}\left(\sigma_{*}\left(X_{p}\right)\right)=g^{-1} \sigma(p)^{-1}\left[\pi_{*} \sigma_{*}\left(X_{p}\right)\right] \\
& =g^{-1} \sigma(p)^{-1}\left(X_{p}\right)=g^{-1} \theta\left(X_{p}\right)
\end{aligned}
$$

where we have used the fact that $\widetilde{\theta} \circ \mathcal{V} \equiv 0$.

The local Cartan connection form $\omega$, in the gauge $\sigma$, is $\omega:=\sigma^{*} \widetilde{\omega}$. We have

$$
\left(\Psi^{*} \widetilde{\omega}\right)\left(X_{(p, g)}+Y_{(p, g)}\right)=\widetilde{\omega}\left(\left(R_{g}\right)_{*} \sigma_{*} X_{p}\right)+\widetilde{\omega}\left(\mathcal{V}\left(\operatorname{Ad}\left(g^{-1}\right)\left(\mu\left(Y_{g}\right)\right)\right) .\right.
$$


We also have

$$
\begin{aligned}
\widetilde{\omega}\left(\left(R_{g}\right)_{*} \sigma_{*} X_{p}\right) & =\left(R_{g}\right)^{*} \widetilde{\omega}\left(\sigma_{*} X_{p}\right)=\operatorname{Ad}\left(g^{-1}\right) \widetilde{\omega}\left(\sigma_{*} X_{p}\right) \\
& =\operatorname{Ad}\left(g^{-1}\right) \sigma^{*} \widetilde{\omega}\left(X_{p}\right)=\operatorname{Ad}\left(g^{-1}\right) \omega\left(X_{p}\right) .
\end{aligned}
$$

and

$$
\widetilde{\omega}\left(\mathcal{V}\left(\operatorname{Ad}\left(g^{-1}\right)\left(\mu\left(Y_{g}\right)\right)\right)=\operatorname{Ad}\left(g^{-1}\right) \mu\left(Y_{g}\right) .\right.
$$

Thus

$$
\left(\Psi^{*} \widetilde{\omega}\right)\left(X_{(p, g)}+Y_{(p, g)}\right)=\operatorname{Ad}\left(g^{-1}\right)\left(\omega\left(X_{p}\right)+\mu\left(Y_{g}\right)\right) .
$$

Now we see that $X_{(p, g)}+Y_{(p, g)}$ is horizontal if and only if

$$
\begin{aligned}
\left(\Psi^{*} \widetilde{\omega}\right) & \left(X_{(p, g)}+Y_{(p, g)}\right)=0 \\
& \Longleftrightarrow \operatorname{Ad}\left(g^{-1}\right)\left(\omega\left(X_{p}\right)+\mu\left(Y_{g}\right)\right)=0 \\
& \Longleftrightarrow \mu\left(Y_{g}\right)=-\omega\left(X_{p}\right) \\
& \Longleftrightarrow Y_{g}=-R\left(\omega\left(X_{p}\right)\right)_{g},
\end{aligned}
$$

where $R\left(\omega\left(X_{p}\right)\right)$ is the right invariant vector field on $G$ determined by $\omega\left(X_{p}\right)$. Lifting to the product $M \times G$, it follows that $Y_{(p, g)}=-R\left(\omega\left(X_{p}\right)\right)_{(p, g)}$. In short, every horizontal vector on $M \times G$ is of the form

$$
X_{(p, g)}-R\left(\omega\left(X_{p}\right)\right)_{(p, g)}
$$

Note that $\left(\Psi^{*} \widetilde{\theta}\right)\left(X_{(p, g)}-R\left(\omega\left(X_{p}\right)\right)_{(p, g)}\right)=g^{-1} \theta\left(X_{p}\right)$, so in order that a horizontal vector $X_{(p, g)}-R\left(\omega\left(X_{p}\right)\right)_{(p, g)}$ correspond to the basic horizontal vector field $H(\xi)$, for $\xi \in \mathbb{R}^{m}$, we must have $g^{-1} \theta\left(X_{p}\right)=\xi$, or $\theta\left(X_{p}\right)=g \xi$, which is the same as $X_{p}=\sigma(p)(g \xi)$. Thus

$$
H(\xi)_{(p, g)}=(\sigma(p)(g \xi))_{(p, g)}-R(\omega(\sigma(p)(g \xi)))_{(p, g)} .
$$

In terms of the frame fields $X_{i}$, we have

$$
\sigma(p)(g \xi)=\sum X_{i}(p)(g \xi)^{i}=\sum X_{i}(p) g_{j}^{i} \xi^{j}
$$

and

$$
\omega(\sigma(p)(g \xi))=\sum \omega\left(X_{i}(p)\right) g_{j}^{i} \xi^{j} .
$$

We conclude that

$$
H(\xi)_{(p, g)}=\sum_{i, j=1}^{m}\left[X_{i}(p, g)-R\left(\omega\left(X_{i}\right)\right)_{(p, g)}\right] g_{j}^{i} \xi^{j}
$$


To simplify the notation a bit, we will use the same notation for a vector field on $M$ (or $G$ ) and its lift to $M \times G$. Then, using $y_{j}^{i}$ to denote the function on $M \times G$ whose value at $(p, g)$ is $g_{j}^{i}$, we write $H(\xi)=\sum\left[X_{i}-R\left(\omega\left(X_{i}\right)\right)\right] y_{j}^{i} \xi^{j}$. If we set $H_{i}:=H\left(e_{i}\right)$, where $\left(e_{1}, \ldots, e_{m}\right)$ is the standard basis on $\mathbb{R}^{m}$, then

$$
H_{j}=\sum\left[X_{i}-R\left(\omega\left(X_{i}\right)\right)\right] y_{j}^{i}
$$

This will serve as the standard form for the horizontal vectors that we use to generate a basis for the subspace of $T_{(p, g)}(M \times G)$ tangent to the reachable set.

\section{Computations on $\boldsymbol{H}^{3}$}

We now specialize to the case where $M=H^{3}$ is the 3 -dimensional Heisenberg group, which is defined to be the group of $3 \times 3$ matrices of the form

$$
\left[\begin{array}{ccc}
1 & a & b \\
0 & 1 & c \\
0 & 0 & 1
\end{array}\right], \quad a, b, c \in \mathbb{R}
$$

under matrix multiplication.

The Lie algebra $\mathfrak{h}$ of $H^{3}$ will be taken to be the tangent space $T_{e} H^{3}$ at the identity element and this is in turn identified with the linear space of matrices of the form

$$
\left[\begin{array}{lll}
0 & a & b \\
0 & 0 & c \\
0 & 0 & 0
\end{array}\right], \quad a, b, c \in \mathbb{R}
$$

so that the Lie Bracket becomes the commutator bracket as usual. We take as a basis for $\mathfrak{h}$ the following three matrices:

$$
X_{1}=\left[\begin{array}{lll}
0 & 1 & 0 \\
0 & 0 & 0 \\
0 & 0 & 0
\end{array}\right], \quad X_{2}=\left[\begin{array}{lll}
0 & 0 & 0 \\
0 & 0 & 1 \\
0 & 0 & 0
\end{array}\right], \quad X_{3}=\left[\begin{array}{lll}
0 & 0 & 1 \\
0 & 0 & 0 \\
0 & 0 & 0
\end{array}\right] .
$$

We have the bracket relations $\left[X_{1}, X_{2}\right]=X_{3},\left[X_{2}, X_{3}\right]=0$, and $\left[X_{1}, X_{3}\right]=$ 0 .

The left invariant vector field associated to any $X \in \mathfrak{h}$ will be denoted by $\widetilde{X}$ and is defined by $\widetilde{X}_{g}:=\left(L_{g}\right)_{*} X$, where $L_{g}$ is left multiplication by $g$. 
We declare that the global left invariant fields $\widetilde{X}_{1}, \widetilde{X}_{2}, \widetilde{X}_{3}$ are everywhere orthonormal, thereby providing $H^{3}$ with a left invariant Riemannian metric. These fields also provide $H^{3}$ with an orientation and the frame bundle has the structure group $\mathrm{SO}(3)$ with Lie algebra $\mathfrak{s o}(3)$ consisting of $3 \times 3$ skewsymmetric matrices. In order to use the trivialization as in the previous section we take $\sigma: H^{3} \rightarrow F\left(H^{3}\right)$ to be given by $\widetilde{X}_{1}, \widetilde{X}_{2}, \widetilde{X}_{3}$.

As a basis $\left[A_{1}, A_{2}, A_{3}\right]$ for $\mathfrak{s o}(3)$ we take

$$
A_{1}=\left[\begin{array}{ccc}
0 & 0 & 0 \\
0 & 0 & 1 \\
0 & -1 & 0
\end{array}\right], A_{2}=\left[\begin{array}{ccc}
0 & 0 & 1 \\
0 & 0 & 0 \\
-1 & 0 & 0
\end{array}\right], \quad A_{3}=\left[\begin{array}{ccc}
0 & 1 & 0 \\
-1 & 0 & 0 \\
0 & 0 & 0
\end{array}\right]
$$

We have the bracket relations

$$
\left[A_{1}, A_{2}\right]=A_{3}, \quad\left[A_{2}, A_{3}\right]=A_{1}, \quad\left[A_{1}, A_{3}\right]=-A_{2} .
$$

We have a global frame field $\left[R\left(A_{1}\right), R\left(A_{2}\right), R\left(A_{3}\right)\right]$ for $\mathrm{SO}(3)$ consisting of the corresponding right invariant fields. Using the same notation for the lifts, we have a global frame field for $H^{3} \times \mathrm{SO}(3)$ :

$$
\mathcal{Z}=\left[\widetilde{X}_{1}, \widetilde{X}_{2}, \widetilde{X}_{3}, R\left(A_{1}\right), R\left(A_{2}\right), R\left(A_{3}\right)\right]
$$

Let $\omega$ be the Cartan connection form corresponding to the metric on $H^{3}$ in the gauge $\sigma$ and let $\theta^{1}, \theta^{2}, \theta^{3}$ be the basis of 1 -forms dual to $\widetilde{X}_{1}, \widetilde{X}_{2}, \widetilde{X}_{3}$. By Cartan's Lemma [10, Vol. II, p. 281, and Chap. 8, in particular p. 336], $\omega$ is the unique skew-symmetric matrix of 1 -forms determined by the equation

$$
d \theta+\omega \wedge \theta=0
$$

where $\theta$ is the column vector $\left[\theta_{1}, \theta_{2}, \theta_{3}\right]^{\top}$ and $d$ is exterior differentiation on each entry. Using the relations $d \theta^{k}\left(\widetilde{X}_{i}, \widetilde{X}_{j}\right)=-\theta^{k}\left(\left[\widetilde{X}_{i}, \widetilde{X}_{j}\right]\right)$, and $\theta^{i}\left(\widetilde{X}_{j}\right)=$ $\delta_{j}^{i}$, Cartan's equations for $\omega=\left(\omega_{j}^{i}\right)$ become

$$
\left[\begin{array}{c}
0 \\
0 \\
-\theta^{1} \wedge \theta^{2}
\end{array}\right]=-\left[\begin{array}{ccc}
0 & \omega_{2}^{1} & \omega_{3}^{1} \\
-\omega_{2}^{1} & 0 & \omega_{3}^{2} \\
-\omega_{3}^{1} & -\omega_{3}^{2} & 0
\end{array}\right]\left[\begin{array}{l}
\theta^{1} \\
\theta^{2} \\
\theta^{3}
\end{array}\right]
$$

with the obvious solution

$$
\omega=\frac{1}{2}\left[\begin{array}{ccc}
0 & \theta^{3} & \theta^{2} \\
-\theta^{3} & 0 & -\theta^{1} \\
-\theta^{2} & \theta^{1} & 0
\end{array}\right]=\frac{1}{2}\left(-A_{1} \theta^{1}+A_{2} \theta^{2}+A_{3} \theta^{3}\right) .
$$


With this determination of $\omega$, we have the basic horizontal vector fields

$$
H_{j}=\sum_{i=1}^{3}\left[X_{i}-R\left(\omega\left(X_{i}\right)\right)\right] y_{j}^{i} .
$$

Notice that given any two dimensional subspace $V_{0}$ of $T_{p} M$ we may choose $g$ so that the tangent bundle projections of $\Psi_{*}\left(H_{1}(p, g)\right)$ and $\Psi_{*}\left(H_{2}(p, g)\right)$ span $V_{0}$. The fields $H_{1}$ and $H_{2}$ span a distribution $E$ on $H^{3} \times \mathrm{SO}(3)$.

A curve in $\gamma:[a, b] \rightarrow H^{3} \times \mathrm{SO}(3)$ that is tangent to $E$ corresponds to an admissible path and the set of frames reachable by curves tangent to $E$ is exactly the leaf through $(p, g)$ of the smallest integrable distribution $E^{*}$ containing $E$. Since we are in the real analytic setting, $E^{*}$ is the distribution spanned by $\mathcal{L}\left(\left\{H_{1}, H_{2}\right\}\right)$, the Lie algebra of vector fields generated by $H_{1}$ and $\mathrm{H}_{2}$.

We will show that this distribution is in fact the whole tangent bundle of $F(M)$ and is spanned by sufficiently many iterated brackets of $H_{1}$ and $\mathrm{H}_{2}$.

Since

$$
\omega\left(X_{1}\right)=-\frac{1}{2} A_{1}, \quad \omega\left(X_{2}\right)=\frac{1}{2} A_{2}, \quad \omega\left(X_{3}\right)=\frac{1}{2} A_{3}
$$

we also have

$$
R\left(\omega\left(X_{1}\right)\right)=-\frac{1}{2} R\left(A_{1}\right), \quad R\left(\omega\left(X_{2}\right)\right)=\frac{1}{2} R\left(A_{2}\right), \quad R\left(\omega\left(X_{3}\right)\right)=\frac{1}{2} R\left(A_{3}\right) .
$$

Thus we have

$$
\begin{aligned}
& H_{1}=y_{1}^{1} \widetilde{X}_{1}+y_{1}^{2} \widetilde{X}_{2}+y_{1}^{3} \widetilde{X}_{3}+\frac{1}{2} y_{1}^{1} R\left(A_{1}\right)-\frac{1}{2} y_{1}^{2} R\left(A_{2}\right)-\frac{1}{2} y_{1}^{3} R\left(A_{3}\right), \\
& H_{2}=y_{2}^{1} \widetilde{X}_{1}+y_{2}^{2} \widetilde{X}_{2}+y_{2}^{3} \widetilde{X}_{3}+\frac{1}{2} y_{2}^{1} R\left(A_{1}\right)-\frac{1}{2} y_{2}^{2} R\left(A_{2}\right)-\frac{1}{2} y_{2}^{3} R\left(A_{3}\right) .
\end{aligned}
$$

Let $W:=\left[H_{1}, H_{2}\right]$. We wish to calculate $W$ as a linear combination of $\mathcal{Z}=\left[\widetilde{X}_{1}, \widetilde{X}_{2}, \widetilde{X}_{3}, R\left(A_{1}\right), R\left(A_{2}\right), R\left(A_{3}\right)\right]$. A few remarks are in order.

1. In our calculation of the brackets of vectors fields we treat vector fields as derivations. The $\widetilde{X}_{k}$ act as zero on the functions $y_{j}^{i}$ while $\left[\widetilde{X}_{k}, R\left(A_{i}\right)\right]=0$.

2. The $R\left(A_{k}\right)$ act on the $y_{j}^{i}$ according to $R\left(A_{k}\right) y_{j}^{i}=\sum a_{l}^{i}(k) y_{j}^{l}$ where $A_{k}=\left[a_{l}^{i}(k)\right]$. 
3. $\left[R\left(A_{i}\right), R\left(A_{j}\right)\right]=-R\left(\left[A_{i}, A_{j}\right]\right)$. The minus sign occurs because the $R\left(A_{i}\right)$ are right invariant fields while bracket is defined in terms of left invariant fields.

The first two observations can be incorporated into a Maple routine in order to calculate $W$ while the third observation allows for an immediate simplification of the rather complicated Maple output ${ }^{1}$. This results in the following expression:

$W=\frac{1}{4}\left(y_{1}^{3} y_{2}^{2}-y_{1}^{2} y_{2}^{3}\right) R\left(A_{1}\right)+\frac{1}{4}\left(y_{1}^{3} y_{2}^{1}-y_{2}^{3} y_{1}^{1}\right) R\left(A_{2}\right)+\frac{3}{4}\left(y_{1}^{1} y_{2}^{2}-y_{2}^{1} y_{1}^{2}\right) R\left(A_{3}\right)$.

Notice that this expression does not involve any of the $\widetilde{X}_{k}$. We may simplify our expression for $W$ even further using the fact that the matrix function $Y=\left[y_{j}^{i}\right]$ takes values in $\mathrm{SO}(3)$. We have

$$
\begin{array}{ll}
\sum_{k=1}^{3} y_{i}^{k} y_{j}^{k}=\delta_{j}^{i}, & \text { because } Y^{\top} Y=I=\left[\delta_{j}^{i}\right], \\
\sum_{k=1}^{3} y_{k}^{i} y_{k}^{j}=\delta_{j}^{i}, & \text { because } Y Y^{\top}=I .
\end{array}
$$

Since $\operatorname{det}(Y)=1$ we have

$$
y_{1}^{1} y_{2}^{2} y_{3}^{3}+y_{1}^{3} y_{2}^{1} y_{3}^{2}+y_{1}^{2} y_{2}^{3} y_{3}^{1}-y_{1}^{2} y_{2}^{1} y_{3}^{3}-y_{1}^{1} y_{2}^{3} y_{3}^{2}-y_{1}^{3} y_{2}^{2} y_{3}^{1}-1=0 .
$$

If we multiply relation (6) by $y_{3}^{3}$, then after a bit of grouping and factoring, we obtain

$$
y_{3}^{3}=\left(y_{3}^{3}\right)^{2}\left(y_{1}^{1} y_{2}^{2}-y_{1}^{2} y_{2}^{1}\right)+y_{3}^{2} y_{3}^{3}\left(y_{1}^{3} y_{2}^{1}-y_{1}^{1} y_{2}^{3}\right)+y_{3}^{1} y_{3}^{3}\left(y_{1}^{2} y_{2}^{3}-y_{1}^{3} y_{2}^{2}\right) .
$$

From (5) we have $y_{3}^{1} y_{3}^{3}=-\left(y_{1}^{1} y_{1}^{3}+y_{2}^{1} y_{2}^{3}\right)$ and $y_{3}^{2} y_{3}^{3}=-\left(y_{1}^{2} y_{1}^{3}+y_{2}^{2} y_{2}^{3}\right)$. Plugging these expressions for $y_{3}^{1} y_{3}^{3}$ and $y_{3}^{2} y_{3}^{3}$ into (7) above, we obtain, after some rearrangement,

$$
y_{3}^{3}=\left(\left(y_{1}^{3}\right)^{2}+\left(y_{2}^{3}\right)^{2}+\left(y_{3}^{3}\right)^{2}\right)\left(y_{1}^{1} y_{2}^{2}-y_{1}^{2} y_{2}^{1}\right) .
$$

Then we use (5) again to see that the first factor on the right hand side above is 1 and thus obtain

$$
y_{3}^{3}=\left(y_{1}^{1} y_{2}^{2}-y_{1}^{2} y_{2}^{1}\right)
$$

\footnotetext{
${ }^{1}$ The routine and the output are available from the authors on request.
} 
In a similar way we obtain

$$
\begin{aligned}
& y_{3}^{2}=\left(y_{1}^{3} y_{2}^{1}-y_{1}^{1} y_{2}^{3}\right), \\
& y_{3}^{1}=\left(y_{1}^{2} y_{2}^{3}-y_{2}^{2} y_{1}^{3}\right) .
\end{aligned}
$$

Using the last three relations (8), (9) and (10), we obtain

$$
W=-\frac{1}{4} y_{3}^{1} R\left(A_{1}\right)+\frac{1}{4} y_{3}^{2} R\left(A_{2}\right)+\frac{3}{4} y_{3}^{3} R\left(A_{3}\right) .
$$

We represent vector fields as the column vectors of their coefficient functions with respect to our global frame $\mathcal{Z}$. So far we have

$$
\begin{aligned}
H_{1} & =\left[y_{1}^{1}, y_{1}^{2}, y_{1}^{3}, \frac{1}{2} y_{1}^{1},-\frac{1}{2} y_{1}^{2},-\frac{1}{2} y_{1}^{3}\right]^{\top}, \\
H_{2} & =\left[y_{2}^{1}, y_{2}^{2}, y_{2}^{3}, \frac{1}{2} y_{2}^{1},-\frac{1}{2} y_{2}^{2},-\frac{1}{2} y_{2}^{3}\right]^{\top}, \\
W & =\left[H_{1}, H_{2}\right]=\left[0,0,0,-\frac{1}{4} y_{3}^{1}, \frac{1}{4} y_{3}^{2}, \frac{3}{4} y_{3}^{3}\right]^{\top} .
\end{aligned}
$$

But we need higher brackets and this is where we need to use Maple again. Our routine uses Maple's ability to simplify with respect to side relations ((5) and (6)). This gives somewhat complicated expressions for the following vector fields obtained by iterated bracketing:

$$
\begin{aligned}
W_{1} & :=\left[H_{1}, W\right] \\
W_{2} & :=\left[H_{2}, W\right] \\
W_{12} & :=\left[W_{1}, W_{2}\right] \\
Y_{1} & :=\left[H_{1}, W_{12}\right] \\
Y_{2} & :=\left[H_{2}, W_{12}\right] .
\end{aligned}
$$

For example, the column vector expression for $Y_{1}$ is

$$
\begin{aligned}
Y_{1}:= & {\left[\frac{9}{8}\left(y_{3}^{3}\right)^{2} y_{1}^{2}-\frac{67}{64} y_{1}^{2} y_{3}^{3}-\frac{3}{8} y_{1}^{3} y_{3}^{2}\left(y_{3}^{3}\right)^{2}-\frac{9}{64} y_{1}^{3} y_{3}^{2},\right.} \\
& -\frac{3}{4}\left(y_{3}^{3}\right)^{4} y_{2}^{2}+\frac{3}{4}\left(y_{3}^{3}\right)^{3} y_{3}^{2} y_{2}^{3}+\frac{13}{16} y_{2}^{2}\left(y_{3}^{3}\right)^{2}-\frac{19}{16} y_{2}^{3} y_{2}^{3} y_{3}^{3}+\frac{9}{64} y_{2}^{2}, \\
& \left.\frac{25}{64} y_{2}^{3}+\frac{15}{8}\left(y_{3}^{3}\right)^{2} y_{2}^{3},-\frac{1}{128} y_{2}^{1},-\frac{1}{128} y_{2}^{2}, \frac{13}{128} y_{2}^{3}\right]^{\top} .
\end{aligned}
$$


Our next goal is to show that $\left[H_{1}, H_{2}, W, W_{1}, W_{2}, W_{12}, Y_{1}, Y_{2}\right]$ spans the whole tangent space at every point $(p, g)$ of $H^{3} \times \mathrm{SO}(3)$. This will show that the reachable set is the entire frame bundle. Since each tangent space in question is 6 dimensional we need only show that at each point, some six of our vectors are linearly independent.

To do this, we will use the Gramian. Recall that if $L=\left[v_{1}, \ldots, v_{k}\right]$ is a list of vectors in $\mathbb{R}^{n}$, then this list is linearly independent if and only if the Gramian

$$
\operatorname{gram}(L)=\operatorname{det}\left(\left\langle v_{i}, v_{j}\right\rangle\right), \quad i, j=1, \ldots, k
$$

is not zero. Here $\langle\cdot, \cdot\rangle$ is the usual inner product on $\mathbb{R}^{n}$.

First consider the list $M_{1}=\left[H_{1}, H_{2}, W, W_{1}, W_{2}, W_{12}\right]$. Using Maple we compute the Gramian of $M_{1}$ :

$$
\operatorname{gram}\left(M_{1}\right)=\frac{1}{4096}\left(y_{3}^{3}\right)^{2}\left(1-6\left(y_{3}^{3}\right)^{2}+11\left(y_{3}^{3}\right)^{4}\right)^{2} .
$$

This is a polynomial in $x=\left(y_{3}^{3}\right)^{2}$. The factor $1-6\left(y_{3}^{3}\right)^{2}+11\left(y_{3}^{3}\right)^{4}$ has discriminant -8 and so $\operatorname{gram}\left(M_{1}\right)$ vanishes exactly when $y_{3}^{3}=0$. Thus, at points where $y_{3}^{3} \neq 0$, the vectors $H_{1}, H_{2}, W, W_{1}, W_{2}, W_{12}$ are linearly independent.

We need to find out what happens where $y_{3}^{3}=0$. We consider the list $M_{2}=\left[H_{1}, H_{2}, W, W_{12}, Y_{1}, Y_{2}\right]$. We obtain

$$
\begin{aligned}
\operatorname{gram}\left(M_{2}\right) & =\frac{9}{268435456}\left(12\left(y_{3}^{3}\right)^{4}-13\left(y_{3}^{3}\right)^{2}+2\right)^{2} \\
& \times\left(24\left(y_{3}^{3}\right)^{8}-24\left(y_{3}^{3}\right)^{6}+11\left(y_{3}^{3}\right)^{4}+10\left(y_{3}^{3}\right)^{2}-1\right)^{2} .
\end{aligned}
$$

When $y_{3}^{3}=0$ this reduces to a nonzero constant and so at these points, $H_{1}, H_{2}, W, W_{12}, Y_{1}, Y_{2}$ are linearly independent.

In conclusion, we see that $\left[H_{1}, H_{2}, W, W_{1}, W_{2}, W_{12}, Y_{1}, Y_{2}\right]$ spans the whole tangent space at each point. Thus, we have $E_{(p, g)}^{*}=T_{(p, g)}\left(H^{3} \times \mathrm{SO}(3)\right)$ at every point $(p, g) \in H^{3} \times \mathrm{SO}^{3}$. Thus, any point in $H^{3} \times \mathrm{SO}(3)$ can be reached from any other point by a curve tangent to $E$.

We need only recall that by the principal bundle trivialization $\Psi$ we may interpret the result in terms of $F\left(H^{3}\right)$ itself with the distribution spanned by the horizontal fields $\Psi_{*} H_{1}, \Psi_{*} H_{2}$. The result can then be expressed in terms of the problem on $M=H^{3}$ as originally stated.

Theorem 5. The distribution defined by the horizontal fields $\Psi_{*} H_{1}, \Psi_{*} H_{2}$ has as reachable set from any point, the entire frame bundle $F\left(H^{3}\right)$. In terms 
of $H^{3}$, this means that if $V_{0}$ and $V_{1}$ are 2-dimensional subspaces of $T_{p_{0}} H^{3}$ and $T_{p_{1}} H^{3}$ respectively, then there is a piecewise smooth curve $\gamma:[0,1] \rightarrow H^{3}$, and a family $\left\{V_{t}\right\}_{t \in[0,1]}$, such that

(i) $\gamma(0)=p_{0}$ and $\gamma(1)=p_{1}$;

(ii) $V_{t}$ is the parallel translate of $V_{0}$ to a subspace of $T_{\gamma(t)} H^{3}$. (iii) $\gamma^{\prime}(t) \in V_{t}$ for all $t \in[0,1]$.

\section{Acknowledgments}

The material in the first three sections was worked out in conversations between Lance Drager and Robert L. Foote. We wish to thank Prof. Foote for many helpful discussions.

The Heisenberg group example was worked out by Jack Follis in a master's thesis directed by the other authors.

Chad Marlowe wrote a master's thesis under the direction of Lance Drager in which he gave a computer simulation of controlling a curve in $H^{3}$ as above, leading us to believe that all points were reachable.

We wish to thank the Referee for helpful comments.

\section{References}

[1] W.-L. Chow, Über systeme von linearen partiellen differtialgleichungen erster ordung, Math. Ann. 117 (1959), 98-105. MR0001880

[2] L. Drager, J. Lee, E. Park, and K. Richardson, Smooth distributions are finitely generated, Annals of Global Analysis and Geometry 41(3) (2012), 357-369. MR2886203

[3] S. Helgason, Integral geometry and Radon transforms, Springer, New York, 2011. MR2743116

[4] R. Hermann, Existence in the large of totally geodesic submanifolds of Riemannian spaces, Bull. Amer. Math. Soc. 66 (1960), 59-61. MR0117682

[5] N. Hicks, A theorem on affine connexions, Illinois J. Math. 3 (1959), 242-254. MR0107875

[6] S. Kobayashi and K. Nomizu, Foundations of differential geometry, Wiley classics library ed., Wiley, New York, 1996.

[7] J. M. Lee, Manifolds and differential geometry, American Mathematical Society, 2009. MR2572292 
[8] P. W. Michor, Topics in differential geometry, Graduate Studies in Mathematics, Vol. 93, American Mathematical Society, Providence, RI, 2008. MR2428390

[9] T. Nagano, Linear differential systems with singularities and an application to transitive Lie algebras, J. Math. Soc. Japan 18 (1966), 398-404. MR0199865

[10] M. Spivak, A comprehensive introduction to differential geometry, 3rd ed., Publish or Perish, Inc, Houston, Tex, 1999.

[11] P. Stefan, Integrability of systems of vector fields, J. London Math. Soc. 21(2) (1980), no. 3, 544-556. MR0577729

[12] H. J. Sussmann, Orbits of families of vector fields and integrability of distributions, Transactions of the American Mathematical Society 180 (1973), 171-188. MR0321133

[13] H. J. Sussmann, Smooth distributions are globally finitely spanned, Analysis and Design of Nonlinear Control Systems, Springer, Berlin, 2008, pp. 3-8. MR2758930

[14] K. Tsukada, Totally geodesic hypersurfaces of naturally reductive homogeneous spaces, Osaka J. Math. 33(3) (1996), 697-707. MR1424680

[15] K. Tsukada, Totally geodesic submanifolds of Riemannian manifolds and curvature-invariant subspaces, Kodai Math. J. 19(3) (1996), 395437. MR1418571

LANCE DRAGER

Department of Mathematics and Statistics

Texas Tech University

LubBOCK, Texas, 79409-1042

USA

E-mail address: lance.drager@ttu.edu

JACK L. FOLLIS

Department of Mathematics

Computer Science And Cooperative Engineering

University of St. Thomas

Houston, TX 77006

USA

E-mail address: follisj@stthom.edu 


\author{
JEFFREY M. LEE \\ Department of Mathematics and Statistics \\ TeXas Tech University \\ LubBock, Texas, 79409-1042 \\ USA \\ E-mail address: jeffrey.lee@ttu.edu
}

ReCeived January 17, 2013 